\title{
Memoria de eventos y personajes históricos y su relación con la identidad nacional en jóvenes universitarios y cadetes de formación castrense en el Perú
}

\author{
Memory of historical events and characters and its relationship with national \\ identity in cadets and university students in Peru
}

\author{
Raysa Robles* \\ Agustín Espinosa**
}

\section{Resumen}

El presente estudio tiene como objetivo describir la relación entre la memoria de eventos y personajes históricos y la identidad nacional peruana a partir de la comparación de respuestas obtenidas de cadetes de una escuela naval $(n=113)$, cadetes de una escuela militar $(n=118)$ y estudiantes de una universidad privada de Lima $(n=115)$. Los resultados muestran una valoración más positiva de la historia y una mayor identificación con el Perú en cadetes de ambas instituciones educativas castrenses, en comparación con los estudiantes universitarios. Estos hallazgos sugieren la relevancia de las narrativas históricas transmitidas en las instituciones de formación académica, sean estas castrenses o cívicas, como fuentes de sesgos de representación de la historia y el impacto que estas representaciones tienen en la identidad nacional.

Palabras clave: identidad nacional, memoria histórica de eventos y personajes, cadetes del

ejército, cadetes de la marina, estudiantes universitarios.

Abstract
This study aims to describe the relationship
among the memory of historical events
and characters and the Peruvian national
identity by comparing responses obtained
from cadets from a naval school $(n=113)$,
cadets from a military school $(n=118)$ and
students from a private university in Lima
$(n=115)$. The results show a more positive
evaluation of the history and greater levels
of identification with Peru in cadets of both
military educational institutions, compared
to university students. These findings
suggest the relevance of historical narratives
transmitted in academically forming
institutions as sources of storytelling biases
according with military or civic traditions,
and how these representations are related to
national identity.

\section{Abstract}

*Pontificia Universidad Católica del Perú. Departamento Académico de Psicología. Mail de contacto: robles.raysa@pucp.pe

** Pontificia Universidad Católica del Perú. Departamento Académico de Psicología. Mail de contacto:_agustin.espinosa@pucp.pe

DOI: https://doi.org/10.46553/RPSI.16.32.2020.p46-67

Fecha de recepción: 25 de marzo de 2020 - Fecha de aceptación: 14 de octubre de 2020 
Keywords: national identity, collective memory of historical events, collective memory of historical characters, university students, navy cadets, army cadets.

\section{Memoria colectiva y memoria de eventos y personajes históricos}

La memoria colectiva hace referencia a un proceso social de reconstrucción del pasado experimentado por los miembros de un grupo, comunidad o sociedad (Halbwachs, 1992). El proceso genera una representación colectiva compartida del pasado, a través del recuerdo de hechos considerados relevantes, e involucra diversos mecanismos sociales de transmisión de información sobre dicho pasado, aun cuando los eventos recordados no hayan sido vividos directamente por todos los miembros del grupo (Herranz \& Basabe, 1999; Páez, Techio, Marques \& Martín Beristain, 2007).

Aunque conceptualmente se pueden encontrar relacionados, existe una diferencia entre la memoria histórica y la memoria colectiva, mientras la primera se basa en los hechos que ocurrieron en el pasado a un conjunto de personas y están documentados, la segunda es el recuerdo propio de un grupo con límites frágiles, basado en las tradiciones y discursos del entorno (Halbwachs, 1980).

Rottenbacher y Espinosa (2010) integran ambas aproximaciones y proponen la idea de una memoria histórica colectiva, por medio de la cual los hechos del pasado se reconstruyen colectivamente, a través de los intercambios e interpretación de la información interior a un grupo y sus instituciones. Se tiende a explicar los cambios históricos de grupo en un continuo, cuyos extremos son un estilo nomotético, que pone énfasis en los procesos histórico-sociales que han afectado al grupo en cuestión, y un estilo personalista, que pone énfasis en los personajes históricos relevantes para dicho grupo. Al respecto, estudios en el Perú han encontrado que la visión personalista tiene un mayor impacto que la visión nomotética en la forma en que se expresan distintos componentes de la identidad nacional (Espinosa, 2011; Rottenbacher \& Espinosa, 2010).

Páez, Marques, Valencia y Vincze (2006) describen la importancia de los mecanismos que influyen en la recordación y en la transmisión de la historia para asegurar los motivos de continuidad y pertenencia de los grupos sociales. Entre los procesos principales se encuentran la omisión, el recuerdo selectivo y la asimilación que contribuyen a una interpretación positiva del pasado del endogrupo, siendo sesgos que facilitan una representación positiva del mismo en el presente (Rottenbacher \& Espinosa, 2010).

Diversos estudios sobre memoria de eventos y personajes históricos a nivel internacional señalan que las personas presentan un "sesgo bélico". Es decir, la libre recordación de eventos relacionados a guerras, conflictos y acciones violentas, seguidos por una recordación de eventos políticos y socioeconómicos que afectan a un endogrupo nacional (Espinosa, 2011; Rottenbacher \& Espinosa, 2010; Liu et al., 2005; Hobsbawm \& Ranger, 1983). Estos estudios también evidencian lo que se denomina un "narcisismo de cohorte", que consiste en considerar como más importantes los hechos que ocurren durante la existencia física del propio grupo etáreo. Estudios en esta línea en el Perú muestran una mayor tasa de recordación de eventos temporalmente 
más próximos a los participantes (Espinosa, 2011). Complementariamente, Liu et al. (2005) encuentran que los sucesos históricos recordados siguen un patrón en "U”, es decir se recuerdan con mayor frecuencia los personajes y eventos que juegan un rol importante en el inicio de la historia de la nación, aunque, por otro lado, también se recuerdan los eventos y personajes más recientes y cercanos en el tiempo.

\section{Identidad nacional y memoria histórica colectiva}

La identidad social ha sido definida como "aquella parte del autoconcepto del individuo que se deriva del reconocimiento de la pertenencia a un grupo o grupos sociales junto con el significado emocional y valorativo de dicha pertenencia" (Tajfel, 1984, p. 292). Una consideración central de la Teoría de la Identidad Social (TIS) es que la pertenencia a un grupo es un estado psicológico (Hogg \& Abrams, 1988; Tajfel, 1982), y va a suponer un proceso que entrelaza el comportamiento individual, la cognición social y diversos fenómenos colectivos (Hogg \& Ridgeway, 2003). El constructo abarca los procesos de identificación con distintos grupos sociales, incluidos aquellos de grandes dimensiones como las naciones, estableciendo que los procesos de identificación nacional van a ser un subtipo específico de identidad social enfocados en el sentido de pertenencia a una nación (Espinosa, 2011; Nigbur \& Cinnirella, 2007; Smith, Giannini, Helkama, Maczynski \& Stumpf, 2005).

Analizando diversas teorías sobre el desarrollo del Self y la identidad, que incluyen la TIS, se ha establecido que existen por lo menos seis motivos identitarios principales que funcionan como guías que, contextualmente, aproximan a las personas a determinados estados identitarios y las alejan de otros. Estos motivos son a) autoestima, b) continuidad, c) pertenencia, d) eficacia, e) significado y f) diferenciación (Vignoles, Golledge, Regalia, Manzi \& Scabini, 2006).

Por otra parte, el estudio de la memoria colectiva establece que ésta está basada en la necesidad de atender motivos o funciones similares a los motivos identitarios descritos (Lyons, 1996). Consistentemente, Rottenbacher y Espinosa (2010) mencionan que la memoria histórica colectiva puede satisfacer dichos motivos identitarios a través del recuerdo de la historia del endogrupo. La memoria histórica colectiva activa estrategias frente a recuerdos que amenazan la autoestima colectiva de individuos altamente identificados a fin de lograr la reconstrucción positiva del pasado del endogrupo (Espinosa, 2011; Rottenbacher \& Espinosa, 2010; Herranz \& Basabe, 1999; Páez et al., 2006).

La articulación entrela identidad social y la memoria histórica colectiva también va a mantener el sentido de continuidad y permitir al individuo construir historias de vida que incluyen progresos y cambios de orientación. Este motivo se complementa con el motivo de diferenciación que busca establecer y mantener la distinción con otros grupos sociales (Billig \& Tajfel, 1973). Al atender a los motivos de continuidad y diferenciación, la memoria histórica colectiva preserva un núcleo estable de representación que permite a los individuos reconocer su identidad social a través del tiempo y definir ciertos rasgos del grupo que permitan distinguirse estereotípicamente de otros (Aguilar, 2002). 
Del mismo modo, la memoria histórica colectiva apuntala el motivo de pertenencia al acentuar los elementos que unen a los miembros del grupo e incluirlos como parte del sí mismo (Espinosa, 2011). Asimismo, mediante la valoración de aspectos positivos de la historia del grupo, la memoria histórica colectiva ayuda a obtener una evaluación positiva de la capacidad de actuar del endogrupo, satisfaciendo adicionalmente el motivo de eficacia (Espinosa, 2011).

Por último, la memoria colectiva permite vincular los elementos del pasado a las expectativas de desarrollo del grupo nacional, satisfaciendo así el motivo de significado al definir metas y objetivos a futuro (Vignoles et al., 2006; Espinosa, 2011).

Teniendo en cuenta que la pertenencia a un grupo condiciona la forma en que los individuos representan y transmiten la historia de su nación (Rottenbacher \& Espinosa, 2010) y que dichas investigaciones se han realizado mayoritariamente en muestras de poblaciones universitarias, se considera relevante conocer cómo se dan estos procesos en jóvenes peruanos con formación castrense, para así comparar las representaciones de la historia que presentan estos con las representaciones expresadas por jóvenes con formación universitaria, para comparar las diferencias o similitudes de los contenidos históricos referidos, la valoración de la historia y la identificación con el endogrupo nacional. Asimismo, se busca analizar la prevalencia de ciertos sesgos que podrían incidir en la forma en que se expresa la memoria histórica colectiva en los grupos referidos.

Esta investigación plantea el estudio de la memoria histórica colectiva rescatando elementos como la valoración y recordación de personajes y eventos históricos, y tiene como objetivo principal explorar las diferencias que existen entre la memoria histórica colectiva, expresada en la recordación y valoración de la historia del Perú y la Identidad Nacional, en jóvenes con formación castrense -marina y ejército- y jóvenes universitarios.

Para abarcar los aspectos que comprende dicho objetivo se plantean los siguientes objetivos específicos:

1. Describir y comparar las variables de identidad nacional: identificación con el Perú, autoestima colectiva, contenidos autoestereotípicos nacionales positivos y negativos, entre cadetes de la escuela naval, cadetes de la escuela militar y estudiantes de una universidad privada de Lima.

2. Describir y comparar los eventos y personajes más relevantes en la muestra general, contrastando el grado de recordación y valoración de los hechos históricos en cada grupo.

A modo de hipótesis se espera encontrar que los cadetes de la escuela naval y los cadetes de la escuela militar se identifiquen en mayor medida con el país y asocien más estereotipos positivos al endogrupo nacional peruano, debido a que las instituciones de formación militar se caracterizan por promover discursos cargados de orgullo nacional, reforzando los autoestereotipos positivos de los connacionales, a diferencia de los estudiantes universitarios, expuestos a discursos ambivalentes o críticos sobre la categoría nacional y su historia. Así también, se espera que los personajes y eventos recordados por cada muestra sean distintos, debido al contexto educativo al cual se encuentran expuestos (véase Agüero, Hurtado \& Flórez, 2005). 


\section{Método}

\section{Participantes}

Los participantes fueron 346 jóvenes peruanos que actualmente residen en la ciudad de Lima. De éstos, 113 (32.7\%) eran cadetes de una escuela naval, $118(34.1 \%)$ eran cadetes de una escuela militar y 115 $(33.2 \%)$ eran estudiantes de una universidad privada de Lima. Los participantes tenían edades entre 15 y 29 años $(M=19.55, D E$ $=2.18$ ).

A continuación, se muestra la caracterización de la muestra respecto a las variables sociodemográficas.

\section{Datos control}

Se registraron datos como el sexo, la edad, el lugar de origen, la institución en la que estudiaban los participantes y el nivel socioeconómico. Este último fue calculado por medio de una escala que indaga sobre el último grado de estudios alcanzado por el jefe de hogar, dónde se atiende el jefe del hogar frente a un problema de salud y los bienes que poseen en casa que son características que discriminan el nivel socioeconómico en la ciudad de Lima (Asociación Peruana de Empresas de Investigación de Mercado [APEIM], 2013).

\section{Medidas de identidad nacional}

Nivel de identificación con el Perú (Espinosa, 2011). Esta medida evalúa la identificación con el Perú a través de un ítem único que consta de la siguiente pregunta: "¿Cuál es su grado de identificación con el Perú?". Esta escala se responde en una escala del 1 al 5, donde 1 era "Nada" y 5 era "Total". Esta pregunta se ha utilizado en investigaciones anteriores al demostrarse su validez de contenido y presentar resultados consistentes con la literatura (Espinosa, 2011; Espinosa, Acosta, Valencia, et al., 2016; Espinosa, Soares-da Silva, Contreras, et al., 2017).

Sub-escala de autoestima colectiva

Tabla 1

Características sociodemográficas de la muestra por grupo

\begin{tabular}{lcccc}
\hline \multicolumn{1}{c}{ Variables } & $\begin{array}{c}\text { Total } \\
N=346\end{array}$ & $\begin{array}{c}\text { Escuela } \\
\text { naval } \\
n=113\end{array}$ & $\begin{array}{c}\text { Escuela } \\
\text { militar } \\
n=118\end{array}$ & $\begin{array}{c}\text { Universidad } \\
n=115\end{array}$ \\
\hline Sexo masculino & $74.3 \%$ & $95.6 \%$ & $100 \%$ & $27 \%$ \\
Origen Lima & $59.5 \%$ & $62.8 \%$ & $37.3 \%$ & $79.1 \%$ \\
Origen Provincia & $40.5 \%$ & $37.2 \%$ & $62.7 \%$ & $20.9 \%$ \\
NSE medio bajo & $2.6 \%$ & $1.9 \%$ & $4.2 \%$ & $1.7 \%$ \\
NSE medio & $27.9 \%$ & $16.8 \%$ & $37.3 \%$ & $28.7 \%$ \\
NSE medio alto & $50 \%$ & $59.8 \%$ & $51.7 \%$ & $39.1 \%$ \\
NSE alto & $19.4 \%$ & $21.5 \%$ & $6.8 \%$ & $30.4 \%$ \\
\hline
\end{tabular}


privada Crocker y Luhtanen (1992). Se utilizó la versión adaptada de la subescala para el contexto peruano (Espinosa, 2011). La escala comprende 4 enunciados que evalúan la relación afectiva con el Perú. El formato de respuesta es de 5 puntos, donde 1 era "Totalmente en desacuerdo" y 5 era "Totalmente de acuerdo". Se ha utilizado la adaptación a la categoría nacional con buenos resultados psicométricos en distintas investigaciones (Espinosa, Acosta, Valencia, et al., 2016; Espinosa, Soares-da Silva, Contreras, et al., 2017) En esta muestra, la escala obtuvo una consistencia interna alta $(\alpha=.81)$

Escala de autoestereotipos colecti-vos peruanos (Espinosa, 2011). Está compuesta por una lista de 24 adjetivos que expresan cómo son los peruanos. Cada adjetivo es valorado en una escala del 1 al 5, donde 1 era Totalmente en desacuerdo y 5 era Totalmente de acuerdo. Espinosa (2011) identifica cuatro dimensiones autoestereotípicas relacionadas con la categoría nacional: peruanos confiables, peruanos desconfiables, peruanos capaces y alegres y peruanos patriotas y solidarios. La medida de adecuación muestral, $K M O=.94$ y el test de esfericidad de Barlett $(p<.01)$, indican que el modelo tiene una estructura factorial apropiada. Para el presente estudio un análisis factorial exploratorio dio como resultado 3 dimensiones que explican en conjunto una varianza total del $51.9 \%$ y se utilizarán para los análisis del presente estudio, a) Peruanos incapaces, con una consistencia interna aceptable $(\alpha=.71)$; b) Peruanos confiables $(\alpha$ $=.86)$; y c) Peruanos no confiables $(\alpha=.89)$. Los ítems que conforman cada dimensión se presentan en la tabla 2 .

\section{Medidas de Memoria Histórica Colectiva}

Cuestionario de mención y valoración de personajes y eventos de la historia del Perú (Liu et al., 2005). Se utilizó la versión adaptada para el caso peruano (Espinosa, 2011). Este instrumento ha sido utilizado en diversos estudios en Perú y Argentina (Rottenbacher \& Espinosa, 2011; Bombelli, Fernández \& Sosa, 2013; Sosa, Bombelli, Fernández, et al., 2013; Sosa, Delfino, Bobowik \& Zubieta, 2016) El cuestionario se presentó con el siguiente enunciado: Imagine que debe dar una clase sobre Historia del Perú. Luego, se pidió a los participantes que mencionen los 10 personajes, positivos o negativos, más importantes de la Historia del Perú y que los evalúen en una escala del 0 al 20, que iba desde Muy negativo (0) hasta Muy positivo (20), esta evaluación estaría alineada con el sistema de calificación utilizado de manera frecuente en la educación secundaria y superior (United Nations Educational Scientific and Cultural Organization [UNESCO], 2010). Luego, se les pidió que siguieran el mismo procedimiento con los 10 eventos que consideraran más importantes de la Historia del Perú. Posteriormente, los personajes y eventos referidos por los participantes fueron categorizados y se creó una variable nominal dicotómica donde $1=$ Recuerda y $0=$ No recuerda en cada personaje y evento identificados. Asimismo, el indicador general de valoración de personajes se obtuvo a partir de un promedio de la suma de las valoraciones de los personajes entre el número de personajes mencionados por el participante, aplicándose el mismo criterio para obtener el indicador general de valoración de eventos. 
52 Memoria de eventos y personajes históricos y su relación con la identidad nacional...

Tabla 2

Análisis factorial exploratorio de la escala de auto-estereotipos colectivos

\begin{tabular}{|c|c|c|c|}
\hline & \multicolumn{3}{|c|}{ Dimensiones } \\
\hline & $\begin{array}{c}\text { Peruanos no } \\
\text { confiables }\end{array}$ & $\begin{array}{l}\text { Peruanos } \\
\text { confiables }\end{array}$ & $\begin{array}{l}\text { Peruanos } \\
\text { incapaces }\end{array}$ \\
\hline Mentirosos & .78 & & \\
\hline Corruptos & .68 & & \\
\hline Ociosos & .66 & & \\
\hline Incumplidos & .66 & & \\
\hline Conformistas & .63 & & \\
\hline Honestos & -.61 & & \\
\hline Atrasados & .57 & & \\
\hline De confianza & -.56 & & \\
\hline No confiables & .56 & & \\
\hline Desarrollados & -.51 & & \\
\hline Individualistas & .47 & & \\
\hline Valientes & & .68 & \\
\hline Exitosos & & .67 & \\
\hline Solidarios & & .67 & \\
\hline Alegres & & .63 & \\
\hline Capaces & & .62 & \\
\hline Patriotas & & .58 & \\
\hline Honrados & & .58 & \\
\hline Cumplidos & & .56 & \\
\hline Trabajadores & & .45 & \\
\hline Incapaces & & & .72 \\
\hline Tristes & & & .61 \\
\hline No quieren a su patria & & & .59 \\
\hline Fracasados & & & .45 \\
\hline
\end{tabular}


Memoria de eventos y personajes históricos y su relación con la identidad nacional...

\section{Procedimiento}

Se solicitaron los permisos necesarios para acceder a las muestras en las escuelas naval, militar y en una universidad privada de la ciudad de Lima. Una vez obtenida dicha autorización, ya en las aulas, se preguntó a las personas su disposición a participar del estudio tras ser informados sobre las condiciones de voluntariedad, anonimato y confidencialidad de los datos que proveyeran. Los participantes del estudio recibieron una batería única de escalas de auto-aplicación. En todas las aplicaciones estuvo presente algún investigador para aclarar dudas o consultas sobre el cuestionario. El tiempo aproximado de la aplicación fue de 20 minutos. Los datos recopilados fueron depurados y procesados a través de estadísticos descriptivos e inferenciales.

\section{Resultados}

Se buscó analizar las diferencias entre en la recordación de personajes, se realizaron

las muestras en los componentes de la identidad nacional evaluados. Para tal fin, se realizaron pruebas ANOVA observándose sistemáticamente que los estudiantes universitarios muestran una representación más negativa de la pertenencia, valoración y definición autoestereotípica del grupo nacional en comparación con los estudiantes de formación castrense.

\section{Recordación y valoración de la historia del Perú}

En primer lugar, se obtuvieron diferencias significativas en la valoración de personajes y eventos de la Historia del Perú entre los grupos evaluados, encontrándose que los estudiantes universitarios tienen una representación más negativa que los estudiantes de formación militar, tanto en la valoración de personajes, como en la valoración de eventos.

Luego, para apreciar las diferencias Tabla 3

Comparación de medias de indicadores de identidad nacional, autoestima colectiva y autoestereotipos colectivos

\begin{tabular}{|c|c|c|c|c|c|c|c|c|c|}
\hline \multirow[b]{2}{*}{ Variable } & \multicolumn{2}{|c|}{ Escuela naval } & \multicolumn{2}{|c|}{ Escuela militar } & \multicolumn{2}{|c|}{ Universidad } & \multirow[b]{2}{*}{$g l$} & \multirow[b]{2}{*}{$\eta^{2}$} & \multirow[b]{2}{*}{$F$} \\
\hline & $n$ & $M(D E)$ & $N$ & $M(D E)$ & $n$ & $M(D E)$ & & & \\
\hline $\begin{array}{l}\text { Id. con el } \\
\text { Perú }\end{array}$ & 113 & $4.73(.48)$ & 118 & $4.70(.48)$ & 115 & $3.83(.70)$ & 2 & .36 & $96.16^{* *}$ \\
\hline $\begin{array}{l}\text { Autoestima } \\
\text { colectiva }\end{array}$ & 112 & $4.92(.28)$ & 118 & $4.86(.31)$ & 114 & $4.20(.83)$ & 2 & .27 & $62.25^{* *}$ \\
\hline $\begin{array}{l}\text { Peruanos } \\
\text { incapaces }\end{array}$ & 111 & $1.61(.72)$ & 117 & $1.44(.58)$ & 115 & $2.19(.59)$ & 2 & .21 & $45.78 * *$ \\
\hline $\begin{array}{l}\text { Peruanos } \\
\text { confiables }\end{array}$ & 111 & $4.21(.59)$ & 117 & $4.39(.43)$ & 114 & $3.53(.48)$ & 2 & .39 & $94.14 * *$ \\
\hline $\begin{array}{l}\text { Peruanos no } \\
\text { confiables }\end{array}$ & 110 & $2.47(.71)$ & 117 & $2.41(.73)$ & 112 & $3.16(.50)$ & 2 & .23 & $45.86^{* *}$ \\
\hline
\end{tabular}

Revista de Psicología. Año 2020. Vol. 16, No 32, pp. 46-67 
54 Memoria de eventos y personajes históricos y su relación con la identidad nacional...

pruebas de Chi-cuadrado que muestran que existen diferencias significativas en la recordación de éstos entre los grupos. Para un mayor conocimiento de los personajes recordados en la muestra, se presenta en la tabla 5 algunos datos de los personajes.

A continuación, se muestra en la tabla
6 las frecuencias y diferencias estadísticas en la recordación de personajes por grupo.

En el caso de la recordación de eventos, se siguió un procedimiento analítico similar al realizado con los personajes. En la tabla 7 se presenta una breve descripción de los eventos más recordados en la muestra.

Tabla 4

Comparación de medias valoración de los personajes y eventos de la historia del Perú

\begin{tabular}{|c|c|c|c|c|c|c|c|c|c|}
\hline \multirow{2}{*}{$\begin{array}{l}\text { Variable } \\
\text { Valoración }\end{array}$} & \multicolumn{2}{|c|}{ Escuela naval } & \multicolumn{2}{|c|}{ Escuela militar } & \multicolumn{2}{|c|}{ Universidad } & \multirow[b]{2}{*}{$g l$} & \multirow[b]{2}{*}{$\eta^{2}$} & \multirow[b]{2}{*}{$F$} \\
\hline & $n$ & $M(D E)$ & $n$ & $M(D E)$ & $n$ & $M(D E)$ & & & \\
\hline Personajes & 113 & $\begin{array}{l}16.39 \\
(3.41)\end{array}$ & 118 & $\begin{array}{l}16.52 \\
(2.69)\end{array}$ & 115 & $\begin{array}{l}12.61 \\
(3.39)\end{array}$ & 2 & .27 & $56.65^{* *}$ \\
\hline Eventos & 113 & $\begin{array}{l}16.44 \\
(3.64)\end{array}$ & 115 & $\begin{array}{l}16.41 \\
(3.85)\end{array}$ & 109 & $\begin{array}{l}11.32 \\
(4.06)\end{array}$ & 2 & .28 & $64.95 * *$ \\
\hline
\end{tabular}

$* * p<.01$

Tabla 5

Resumen de personajes de la Historia del Perú

Miguel Grau

Marino peruano que combatió en la Guerra contra España (1864-1866) y comandó el buque Huáscar en la Guerra con Chile (1879) donde murió en combate (Durand, 2005b).

Político peruano, fue presidente de la República (1990-2000). Realizó un Golpe de Estado para disolver el Congreso de la $\begin{array}{ll}\text { Alberto Fujimori } & \text { de poder. Se reeligió en 1995, incurriendo en varios actos } \\ & \text { de corrupción que incluían al Poder Judicial, los medios de } \\ \text { comunicación y la complicidad con líderes militares (Quiroz, } & \\ & \text { 2013). } \\ & \text { Militar que combatió en la Guerra con Chile en las batallas de } \\ \text { Francisco Bolognesi } & \begin{array}{l}\text { San Francisco y Tarapacá. Se le encargó la comandancia general } \\ \text { en la batalla de Arica, muriendo en la lucha el } 7 \text { de junio de 1980 } \\ \text { (Durand, 2005b) }\end{array}\end{array}$ República en 1992, controlando las principales instituciones

Continua en página 55 


\section{Continua en página 54}

\begin{tabular}{ll}
\hline & Político y abogado peruano. Militante de la Alianza Popular \\
& Revolucionaria Americana (APRA). Presidente de la República \\
en los periodos de 1985-1990 y desde el 2006-2011. Investigado \\
por múltiples actos de corrupción (Quiroz, 2013). \\
Militar que combatió en la Guerra con Chile (1879-1883), fue \\
Presidente del Perú en dos ocasiones (1886-1890 y 1894-1895). \\
Inició su carrera militar en 1854. Lideró la campaña de La Breña \\
en 1883 (Durand, 2005b).
\end{tabular}

José de San Martín

Militar argentino que luchó por la Independencia de Argentina, Perú y Chile (Durand, 2005a).

Túpac Amaru II

Cacique de Surimana, Pampamarca y Tungasuca, dedicado al comercio y a la arriería. Protestó contra las reformas tributarias que impuso el virrey Areche en 1780, organizando una gran rebelión anticolonial. Fue capturado y descuartizado en la plaza de Cuzco en 1781 (Durand, 2005a).

Ex - catedrático de filosofía. Líder de la organización terrorista

Abimael Guzmán

y subversiva Sendero Luminoso durante el Conflicto Armado Interno en el Perú (CVR, 2004).

Militar y político peruano. Fue presidente del Perú en dos ocasiones (1845-1851 y 1855-1862). Realizó importantes Ramón Castilla reformas políticas como la abolición de la esclavitud y la abolición del tributo indígena. Es patrón del Arma de Caballería del Ejército Peruano (Durand, 2005a).

Militar y político peruano. Fue Jefe de las Fuerzas Armadas del Juan Velasco Alvarado Perú, y depuso al Presidente Fernando Beláunde, ocupando la presidencia desde 1968 hasta 1975 (Quiroz, 2013).

Militar venezolano. Lideró la liberación de Venezuela y participó

Simón Bolívar en la lucha de la independencia de Colombia, Ecuador y Perú (Durand, 2005a).

\section{Continua en página 56}


Continua en página 55

José A. Quiñones

José Olaya

Vladimiro Montesinos

Mario Vargas Llosa

Alejandro Toledo

Alfonso Ugarte

Francisco Pizarro
Aviador de guerra peruano, que falleció en una misión aérea durante la Guerra de 1941 (Ministerio de Defensa, 2014b).

Ciudadano peruano prócer de la independencia del Perú (Durand, 2005a).

Militar y político peruano, fue principal asesor en el gobierno de Alberto Fujimori, teniendo a cargo la seguridad del Estado y la Dirección del Servicio de Inteligencia Nacional (SIN). Ha sido acusado y sentenciado por delitos de genocidio, tortura, homicidio calificado y corrupción (Quiroz, 2013).

Escritor peruano, crítico literario, ganó el Premio Nobel de Literatura en el 2010. Contendor político de Alberto Fujimori en las elecciones de 1990 (Quiroz, 2013).

Político peruano, economista y catedrático. Fue elegido presidente de la República en el periodo del 2001 al 2006 (Quiroz, 2013).

Militar peruano y comerciante. Participó en la batalla de San Francisco, Tarapacá. En 1880 combatió en la batalla de Arica junto al Coronel Bolognesi, donde falleció (Durand, 2005b).

Español que logró conquistar el Imperio incaico e inicia la colonización del territorio peruano (Hampe, 2005).
A continuación, se muestran en la tabla 8 las frecuencias y diferencias estadísticas en la recordación de eventos por grupo educativo.

En general, se reporta una tendencia de los cadetes de las escuelas naval y militar a recordar con mayor frecuencia combates, batallas y operaciones militares específicas, con un sesgo particular según el arma de pertenencia. Así, los cadetes navales reportan 
Memoria de eventos y personajes históricos y su relación con la identidad nacional...

Tabla 6

Comparación de porcentajes de recordación de Personajes de la Historia del Perú

\begin{tabular}{lcccccccc}
\hline & \multicolumn{7}{c}{ Escuela naval } & \multicolumn{2}{c}{ Escuela militar } & \multicolumn{2}{c}{ Universidad } & \\
\cline { 2 - 8 } Personajes & $f_{\text {total }}$ & $\%$ & $f$ & $\%$ & $f$ & $\%$ & $f$ & $\chi 2$ \\
\hline Miguel Grau & 226 & $96.50 \%$ & 109 & $42.40 \%$ & 50 & $58.30 \%$ & 67 & $78.33^{* *}$ \\
Alberto Fujimori & 175 & $41.60 \%$ & 47 & $40.70 \%$ & 48 & $69.60 \%$ & 80 & $24.86^{* *}$ \\
Francisco Bolognesi & 171 & $57.50 \%$ & 65 & $77.10 \%$ & 91 & $13.00 \%$ & 15 & $100.06^{* *}$ \\
Alan García & 118 & $16.80 \%$ & 19 & $33.10 \%$ & 39 & $52.20 \%$ & 60 & $31.80^{* *}$ \\
Andrés A. Cáceres & 118 & $24.80 \%$ & 28 & $66.90 \%$ & 79 & $9.60 \%$ & 11 & $91.83^{* *}$ \\
José de San Martín & 116 & $37.20 \%$ & 42 & $28.80 \%$ & 34 & $34.80 \%$ & 40 & 1.93 \\
Túpac Amaru II & 75 & $14.20 \%$ & 16 & $11.90 \%$ & 14 & $39.10 \%$ & 45 & $31.09^{* *}$ \\
Abimael Guzmán & 74 & $23.00 \%$ & 26 & $13.60 \%$ & 16 & $27.80 \%$ & 32 & $7.31^{*}$ \\
Ramón Castilla & 73 & $16.80 \%$ & 19 & $33.10 \%$ & 39 & $13.00 \%$ & 15 & $15.86^{* *}$ \\
J. Velasco Alvarado & 65 & $3.50 \%$ & 4 & $32.20 \%$ & 38 & $20.00 \%$ & 23 & $31.25^{* *}$ \\
Simón Bolívar & 57 & $9.70 \%$ & 11 & $17.80 \%$ & 21 & $21.70 \%$ & 25 & $6.20^{*}$ \\
José A. Quiñones & 57 & $39.80 \%$ & 45 & $10.20 \%$ & 12 & $0.00 \%$ & 0 & $70.86^{* *}$ \\
José Olaya & 55 & $12.40 \%$ & 14 & $27.10 \%$ & 32 & $7.80 \%$ & 9 & $17.76^{* *}$ \\
Vladimiro & & & & & & & & \\
Montesinos & 54 & $17.70 \%$ & 20 & $11.90 \%$ & 14 & $17.40 \%$ & 20 & 1.91 \\
Mario Vargas Llosa & 49 & $10.60 \%$ & 12 & $12.70 \%$ & 15 & $19.10 \%$ & 22 & 3.71 \\
Alejandro Toledo & 44 & $6.20 \%$ & 7 & $13.60 \%$ & 16 & $18.30 \%$ & 21 & $7.59^{*}$ \\
Alfonso Ugarte & 33 & $12.40 \%$ & 14 & $11.90 \%$ & 14 & $4.30 \%$ & 5 & 5.40 \\
Francisco Pizarro & 33 & $9.70 \%$ & 11 & $9.30 \%$ & 11 & $9.60 \%$ & 11 & 0.01 \\
\hline * $p$ × & & & & & & & &
\end{tabular}

$*_{p}<.05, * * p<.01$

más hechos vinculados a su arma, y lo propio hacen los cadetes del ejército. Por otra parte, se aprecia en los estudiantes universitarios una tendencia a recordar eventos políticos y procesos históricos que abarcan grandes periodos de tiempo, recordando más que los jóvenes con formación castrense la Guerra con Chile como un proceso general, en contraste con la mayor recordación de aspectos específicos de esta guerra -batallas y combates- por parte de los jóvenes cadetes navales y del ejército. 


\section{Tabla 7}

Resumen de eventos de la Historia del Perú

Periodo de guerras de independencia que inician en 1780 con levantamientos contra el dominio español, hasta las campañas libertadoras dirigidas por José de San Martín y Simón Bolívar que Independencia del Perú permiten la declaración de la independencia el 28 de julio de 1821. Sin embargo, la derrota final de las tropas realistas y la aceptación de la libertad se da tras la Batalla de Ayacucho en 1824 (Durand, 2005a).

Operación Chavín de Ocurrió el 22 de abril de 1997. Operación militar que tenía como Huántar objetivo liberar a 71 rehenes de la residencia del embajador de Japón en el Perú, de manos del grupo subversivo MRTA (CVR 2003).

Se inicia debido a causas económicas, geopolíticas y territoriales, Guerra con Chile (1879- culminó con la ratificación del Tratado de Ancón que confirmaba 1884)

la retirada del ejército chileno manteniendo parte del territorio peruano en su poder (Durand, 2005b).

Combate de Angamos ( 8 Combate naval de la Guerra con Chile, donde fallece Miguel Grau de octubre de 1879) (Durand, 2005b).

Periodo en el que se llevaron a cabo numerosos atentados terroristas, Época de terrorismo (1980-2000) matanzas, secuestros por los grupos subversivos PCP- Sendero Luminoso y Movimiento Revolucionario Túpac Amaru, así como episodios de torturas y violación de derechos humanos por parte de las Fuerzas Armadas. (CVR, 2004)

Combate de 2 de Mayo Combate naval que culmina con las pretensiones españolas de (1866) recuperar sus colonias en América (Durand, 2005b).

Batalla de Ayacucho (9 de diciembre de 1824)

Batalla definitiva en la guerra de independencia que ganó el ejército peruano y colombiano al mando del general Sucre, sobre el ejército español (Durand, 2005a).

Combate de Iquique (21 de mayo de 1879)

Combate naval de Guerra con Chile (Durand, 2005b).

Conflicto del Alto Cenepa (1995)

Conflicto fronterizo entre Perú y Ecuador. Este conflicto finalizó con un arreglo pacífico entre ambos países (Ministerio de Defensa, 2014a).

Conquista y colonia del Perú

Periodo que inicia desde la rendición del Imperio Incaico en 1532 hasta la colonización española y el establecimiento del virreinato (Hampe, 2005). 
Memoria de eventos y personajes históricos y su relación con la identidad nacional...

Tabla 8

Comparación de porcentajes de recordación de Eventos de la Historia del Perú

\begin{tabular}{|c|c|c|c|c|c|c|c|c|}
\hline \multirow[b]{2}{*}{ Evento } & \multirow[b]{2}{*}{$f_{\text {Total }}$} & \multicolumn{2}{|c|}{ Escuela naval } & \multicolumn{2}{|c|}{ Escuela militar } & \multicolumn{2}{|c|}{ Universidad } & \multirow[b]{2}{*}{$\chi^{2}$} \\
\hline & & $\%$ & $f$ & $\%$ & $f$ & $\%$ & $f$ & \\
\hline Independencia del Perú & 180 & $48.70 \%$ & 55 & $42.40 \%$ & 50 & $65.20 \%$ & 75 & $12.93 * *$ \\
\hline $\begin{array}{l}\text { Operación Chavín de } \\
\text { Huántar }\end{array}$ & 119 & $27.40 \%$ & 31 & $72.90 \%$ & 86 & $1.70 \%$ & 2 & $134.24 * *$ \\
\hline Guerra con Chile & 118 & $23.00 \%$ & 26 & $29.70 \%$ & 35 & $49.60 \%$ & 57 & $19.46 * *$ \\
\hline Combate de Angamos & 114 & $70.80 \%$ & 80 & $14.40 \%$ & 17 & $14.80 \%$ & 17 & $108.81 * *$ \\
\hline Época del terrorismo & 83 & $15.90 \%$ & 18 & $12.70 \%$ & 15 & $43.50 \%$ & 50 & $36.21 * *$ \\
\hline Combate de 2 de Mayo & 68 & $37.20 \%$ & 42 & $16.10 \%$ & 19 & $6.10 \%$ & 7 & $36.30 * *$ \\
\hline Batalla de Ayacucho & 64 & $15.90 \%$ & 18 & $31.40 \%$ & 37 & $7.80 \%$ & 9 & $22.12 * *$ \\
\hline Combate de Iquique & 63 & $54.00 \%$ & 61 & $0.80 \%$ & 1 & $0.90 \%$ & 1 & $144.20 * *$ \\
\hline $\begin{array}{l}\text { Conflicto del Alto } \\
\text { Cenepa }\end{array}$ & 61 & $10.60 \%$ & 12 & $41.50 \%$ & 49 & $0.00 \%$ & 0 & $74.84 * *$ \\
\hline $\begin{array}{l}\text { Conquista y colonia del } \\
\text { Perú }\end{array}$ & 45 & $10.60 \%$ & 12 & $10.20 \%$ & 12 & $18.30 \%$ & 21 & 4.22 \\
\hline
\end{tabular}

\section{Discusión}

El objetivo del presente estudio fue describir y comparar la identidad nacional entre cadetes de la escuela naval, cadetes de la escuela militar y estudiantes de una universidad privada de Lima, así como los eventos y personajes más relevantes en la muestra general, contrastando el grado de recordación y valoración de los hechos históricos en cada grupo. Los hallazgos de este estudio permiten analizar los distintos sesgos en la recordación e identidad de la historia, así como las estrategias que se construyen para defender la imagen del endogrupo. En este sentido, cuanto más jerárquico es el grupo, las estrategias para proteger la identidad son más efectivas a nivel estadístico y de discurso. Es importante analizar el costo de esas defensas para la construcción de la identidad y la historia, ya que esto determina cómo los grupos recuerdan y se relacionan con los demás.

\section{Identidad nacional, autoestima colectiva y autoestereotipos nacionales}

Las diferencias obtenidas en las variables de identidad pueden explicarse por la formación que se recibe en las escuelas de formación castrense, caracterizadas por la presencia de rituales, símbolos y 
celebraciones que exaltan la identificación con la nación y con la institución a la cual pertenece el cadete (Agüero et al., 2005). El énfasis en imágenes de cohesión nacional promueve la construcción y continuidad de la identidad social (González Castro, 2006). Por ello, al menos a nivel de discurso, los símbolos propios de cada escuela proveen a los cadetes de una identidad nacional ligada a la institución y diferenciada de lo civil, siendo la esfera civil relacionada a una identidad ambivalente (Espinosa, 2011). Asimismo, es esperable que los valores relacionados con la vida castrense -lealtad, camaradería, confianza en el compañero- refuercen los atributos positivos de la categoría nacional y mitiguen los aspectos negativos asociados a la categoría nacional. En cambio, la muestra universitaria tiene acceso a información ambivalente sobre peruanidad por estar más expuestos a los medios de información y a material crítico para incentivar la reflexión y discusión de la realidad (Agüero et al., 2005).

\section{Recordación y valoración de la historia del Perú}

Una valoración más positiva de la Historia del Perú en los grupos con formación castrense puede explicarse por la visión de la historia y el acceso a la información que estos grupos tienen. Los cursos de historia en las instituciones militares exponen la orientación de la escuela -Historia Naval o Historia Militar-, reforzando así elementos, tácticas y estrategias características de cada arma, lo cual se relaciona al sesgo por institución presente en las narrativas, reforzando en cada caso el dominio y poderío peruano por mar o por tierra. Así, se aprecia un sesgo endogrupal en los procesos de recordación y valoración de eventos que son distintivos y por ende considerados como más importantes para la definición de los atributos del propio grupo.

Por otro lado, los textos de historia a los que tienen acceso los estudiantes universitarios resaltan aspectos civiles, como las acciones políticas de determinadas épocas, la situación social y económica del país, presentando un análisis más crítico y generalista de la historia. Parece entonces que los universitarios están en contacto con discursos históricos ambivalentes, que por un lado presentan una nación desintegrada, con estratos diferenciados y grandes crisis políticas y económicas, y por el otro, una nación incipiente que gracias a grandes figuras nacionales y extranjeras comenzaba a forjar su identidad, lo cual también explicaría la representación más negativa de la valoración de la historia en contraste con los grupos de formación militar (Cotler, 2005; Comisión de la Verdad y Reconciliación [CVR], 2004; Portocarrero \& Oliart, 1989).

Los resultados también corroboran la presencia de un sesgo bélico en la muestra, exacerbado por la participación de jóvenes con formación militar. Lo anterior de manera general confirma la dominancia de la violencia como una narrativa importante debido a la impronta emocional que tienen estos eventos y figuras (Espinosa, 2011; González Castro, 2006; Bobowik et al., 2010; Liu et al., 2005; Pennebaker, Páez \& Deschamps, 2006; Rottenbacher \& Espinosa, 2010). Adicionalmente, se aprecia a nivel descriptivo la predominancia de personajes masculinos relacionados a eventos militares y políticos, los cuáles en su mayoría pertenecen a grupos blancos y mestizos de alto estatus social (Espinosa, 2011; Rottenbacher \& Espinosa, 2010). Esto 
último da cuenta que las representaciones de la Historia del Perú, en las muestras estudiadas tiene además un fuerte sesgo de género y raza, con lo cual es factible afirmar que el pasado en un país estamental como el Perú, es masculino y racializado hacia lo blanco-mestizo, en una clara representación de los grupos que detentan el poder.

Se menciona la existencia de un sesgo socio-céntrico (Pennebaker et al., 2006), referido a enfatizar los sucesos importantes para la nación, especialmente aquellos cambios sociales más profundos (Sibley, Liu, Duckitt\&Khan, 2008) comola Independencia del Perú, la abolición de la esclavitud o la época del terrorismo. Específicamente, se aprecia un sesgo por institución, existiendo una mayor recordación de personajes y eventos específicos relacionados a la historia naval en caso de los cadetes de la escuela naval -Martín Jorge Guise, Diego Ferré, Elías Aguirre, Combate de Iquique, Combate de Abtao, Combate de 2 de Mayo-, una mayor referencia a acontecimientos de la historia militar como batallas y operaciones terrestres en cadetes de la escuela militar -Operación Chavín de Huántar, Conflicto del Alto Cenepa, intervención en los conflictos sociales- y un mayor recuerdo de sucesos políticos generales e importantes para la consolidación de la república y el establecimiento de la democracia en el caso de los universitarios -Juan Velasco Alvarado, Ramón Castilla, Conquista y colonia del Perú, Abolición de la esclavitud-. Estos resultados son esperables por el rol que juega el énfasis de dichos sucesos históricos en la diferenciación de los aspectos que definen por una parte la identidad nacional y por otra la identidad de rol, ya sea militar o civil (Agüero et al., 2005; Liu \& László, 2007).
En general, se aprecia en las instituciones castrenses un menor recuerdo y una valoración más benevolente de sucesos que se evalúan como negativos, comparado al grupo universitario. Por el contrario, los estudiantes universitarios recuerdan más personajes y eventos consensualmente evaluados como negativos -Guerra con Chile, Época del terrorismo y Primer gobierno de Alan García, Alberto Fujimori, Alejandro Toledo, Abimael Guzmán- y valoran menos los personajes y eventos consensualmente positivos -Miguel Grau, Francisco Bolognesi, Andrés A. Cáceres, San Martín, Túpac Amaru II, Independencia del Perú, Operación Chavín de HuántarEste resultado se explica por la defensa de la identidad que se refleja en un recuerdo selectivo de los sucesos positivos y el olvido o resignificación de los sucesos negativos (Espinosa, 2011; Herranz \& Basabe, 1999; Hirst \& Manier, 2008; Páez et al., 2006; Rottenbacher \& Espinosa, 2010). Este sesgo presente en los alumnos de instituciones castrenses puede deberse a la transmisión jerárquica de la información con un sentido poco crítico, con la finalidad de mantener una imagen positiva del endogrupo (Agüero et al., 2005).

\section{Relaciones entre memoria e identificación con el Perú}

En esta misma línea, se explica el importante rol que juegan algunos personajes y eventos históricos para la defensa de la identidad nacional.

Miguel Grau, es el personaje con mayor valoración y recordación en la muestra, juega un rol fundamental en el caso de los cadetes de la escuela naval, no solo por 
su desempeño táctico en la Guerra con Chile, sino por lo que representa como peruano al afianzar la representación del endogrupo como moral. Así también, Francisco Bolognesi y Andrés Avelino Cáceres son referentes importantes de peruano para los cadetes de la escuela militar, debido a su desempeño militar en las batallas de Arica, Tarapacá y las batallas en el sur del país (Chávez, 2010).

Sucesos como la Independencia y la Guerra con Chile son narrativas importantes para el peruano, "más allá de que se hayan perdido o ganado las batallas, se forjaron héroes patrios y símbolos positivos de la nacionalidad" (Contreras, 2006). Por otro lado, la Guerra con Chile se valoraría negativamente porque muestra la fragilidad de la identidad nacional, la corrupción de la clase política, una efímera bonanza económica y las marcadas diferencias sociales en nuestro país (Chávez, 2010). Sin embargo, a pesar del fracaso de la Guerra del Pacífico, se aprecia claramente en los jóvenes con formación castrense una resignificación positiva de la misma a partir de eventos específicos como el Combate de Angamos o las Batallas de Arica y Tarapacá, donde a pesar de la derrota hay lecciones y aspectos de valentía, sacrificio y moralidad que parecen rescatarse y apuntalar una imagen positiva de los miembros del endogrupo nacional.

Los personajes y eventos negativos para el país -Alberto Fujimori, Alejandro Toledo, Abimael Guzmán, Alan García, Época del terrorismo, Guerra con Chile, Primer gobierno de Alan García- son más recordados por los universitarios, indicando un olvido casi institucionalizado de la importancia de los sucesos negativos en los cadetes de la escuela naval y la escuela militar (González Castro, 2006; Páez et al., 2006; Pennebaker et al., 2006), que responde a una función de protección de la soberanía y orgullo nacional que se promueve incluso desde relatos de los historiadores (Chávez, 2010; Espinosa, 2011). Los personajes mencionados reforzarían los autoestereotipos negativos de peruano como corruptos, no confiables, deshonestos e incapaces consolidados en las figuras políticas del país. El olvido o menor recordación de los eventos de violencia política en el país en cadetes de las escuelas naval y militar, puede explicarse por el sentimiento de culpa colectiva que generaría la participación de las Fuerzas Armadas en esta época, en la que se cometieron abusos "justificados" por la seguridad y el orden del país (CVR, 2004; Theidon, 2009). Anteriores estudios han encontrado que cuando se presenta un evento negativo que es responsabilidad de los miembros del endogrupo, se reduce la credibilidad asignada al mensaje y se produce un menor recuerdo de este (Páez et al., 2006).

\section{Conclusiones y recomendaciones}

En conclusión, existe una valoración más positiva de la historia en cadetes de las escuelas naval y militar, así como un sesgo de recordación referido a la institución a la cual pertenecen. Esto puede deberse a los diferentes tipos de información a la que tienen acceso los cadetes y los universitarios, y al rol que juega la memoria colectiva en el mantenimiento y protección de la identidad nacional. Estos hallazgos demuestran la relevancia de los discursos de la historia nacional reforzados en las instituciones 
de formación académica, y su relación con la identidad nacional. Por otro lado, se evidencia una mayor identificación con el endogrupo nacional y una valoración más positiva de peruano en los grupos de formación castrense. Según lo encontrado en investigaciones similares en nuestro país una mayor identificación con el endogrupo nacional estaría relacionado a los mayores niveles de autoritarismo en la muestra peruana (Valencia, Espinosa, Jiménez \& Romero, 2018).

Es necesario reflexionar acerca del desempeño que tuvieron personajes como Miguel Grau, Francisco Bolognesi o Alfonso Ugarte en la historia del Perú, que por solidaridad con la nación y en aras de su defensa, ofrendaron sus vidas enfrentándose a tropas superiores, incluso ante el abandono de la corrupta e ineficiente clase política del país (Faverón, 2013). No se pretende con esto exacerbar el nacionalismo, que conllevaría a repetir errores en la historia, sino rescatar el sacrificio de estos peruanos como una reserva moral importante en la nación $y$ promover su conocimiento y valoración también en espacios civiles, esto permitiría la construcción de una identidad positiva $\mathrm{y}$ una cultura de paz que forme una conciencia nacional de rechazo a la violencia.

Finalmente, incluir en el currículo de educación básica y superior la enseñanza de las crisis políticas, las dinámicas de corrupción en los gobiernos y el Conflicto Armado Interno, es importante para fomentar la capacidad crítica de los estudiantes, pero se percibe la ausencia de estas temáticas en el aula debido a un clima institucional poco propicio, los temores asociados a la forma en que deben abordarlas y la poca vinculación de estas temáticas a la formación ciudadana (Ucceli, Agüero, Pease, Portugal $\&$ Del Pino, 2013). Para iniciar un proceso de reconciliación y perdón en la sociedad peruana es necesario un reconocimiento desde las Fuerzas Armadas de la violencia generada en esta época y compromiso desde la formación militar que se brinda a los cadetes para asumir una actitud crítica de lo acontecido. Un proceso de reflexión explícito sobre la época de violencia política reduce la prevalencia de actitudes propensas a la violencia y reduce las ideologías conservadoras, autoritarias y de dominancia social (Espinosa, Cueto \& Páez, 2019). En este sentido, las personas que no han sido directamente afectadas por este período de violencia que tienen acceso a esta información, estarán preparadas para brindar soporte y rechazar cualquier situación de injusticia en el futuro (Espinosa et al., 2019).

\section{Referencias}

Agüero, F. (2005). Educación militar en democracia: aproximaciones al proceso educativo militar. Lima: Instituto de Defensa Legal.
Aguilar, M. (2002). Fragmentos de la Memoria colectiva. Athenea digital, 2, 1-12. Recuperado de http://ddd. uab.es/pub/athdig/15788946n2a5. 
pdf

Asociación Peruana de Empresas de Investigación de Mercado [APEIM](2013).Informe de Niveles socioeconómicos 2013. http://apeim.com.pe/wp-content/ up lo ads/2019/11/ A P E I M NSE-2013.pdf

Billig, M. \& Tajfel, H. (1973). Similarity and categorization in intergroup behaviour. European Journal of Social Psychology, 3, 27-52.

Bobowik, M., Páez, D., Liu, J., Espinosa, A., Techio, E., Zubieta, E. \& Cabechinas, R. (2010). Beliefs about history, the meaning of historical events and culture of war. Revista de Psicología, 28(1), 111146.

Bombelli, J., Fernández, O. \& Sosa, F.(2013).Representaciones sociales de la historia argentina y género. Un estudio con estudiantes militares. Boletín de Psicología, 108, 71-89.

Chávez, J. M. (2010). La Guerra del Pacífico y la idea de Nación. Lima: La Casa del Libro Viejo.

Comisión de la Verdad y Reconciliación. (2003). Informe Final de la Comisión de la Verdad y Reconciliación Vol. VI. Lima: CVR.

Comisión de Entrega de la CVR. (2004). Hatun Willakuy. Versión abreviada del Informe Final de la Comisión de la Verdad y Reconciliación. Lima: Comisión de Entrega de la CVR.

Contreras, C. (2006, 29 de agosto). Memoria y verdad sobre la guerra con Chile. La Republica On Line. Recuperado de https://larepublica. $\mathrm{pe} /$ politica/273508-memoria-y- verdad-sobre-la-guerra-con-chile/

Cotler, J. (2005). Clases, Estado y Nación en el Perú. (3era Edición). Lima: IEP.

Crocker, J. \& Luhtanen, R. (1992). A collective self-esteem scale: selfevaluation of one's social identity. Personality and Social Psychology Bulletin, 18, 302-318.

Durand, L. (2005a). El nacimiento republicano: Las guerras de la independencia y el sueño de la Confederación (7-141). En C. Arias (Editor). Compendio Histórico del Perú (Vol. 6., $3^{\text {a }}$ edición). Lima: Milla Batres.

Durand, L. (2005b). La era del guano y la guerra del Pacífico (7-141). En C. Arias (Editor) Compendio Histórico del Perú. (Vol. 7, 3ª edición). Lima: Milla Batres,

Espinosa, A. (2011). Estudios sobre identidad nacional en el Perú y sus correlatos psicológicos, sociales y culturales (Tesis doctoral), Universidad del País Vasco, San Sebastián, España.

Espinosa, A., Acosta, Y., Valencia, J., Vera, A., Soares da Silva, A., Romero, J. C. \& Beramendi, M. (2016). Calidez, competencia, moralidadynacionalismoidealcomo dimensiones autoestereotípicas del autoconcepto nacional en seis países de Latinoamérica. Avances en Psicología Latinoamericana, 34(2), 395-413. doi: http://dx.doi. org/10.12804/apl34.2.2016.12

Espinosa, A., Soares-da Silva A., Contreras, C., Cueto, R., García A., Ortolano, F., Valencia, J., \& Vera, A. (2017). Identidad nacional y sus relaciones con la ideología y el bienestar 
Memoria de eventos y personajes históricos y su relación con la identidad nacional...

en cinco países de América Latina. Avances en Psicología Latinoamericana, 35(2), 351-374. doi:http://dx.doi.org/10.12804/ revistas.urosario.edu.co/apl/a.3765

Espinosa, A., Cueto, R.M., Páez, D. (2019). Reflecting upon the years of political violence in Peru. An experimental study to promote a cultural of peace in higher education. Journal of Prevention \& Intervention in the Community. DOI: 10.1080/10852352.2019.1664710

Faverón, G. (2013, 27 de julio). Los pendejos y los cojudos (a propósito de una obra teatral de Rocío Tovar) (Blog) Recuperado de http://gustavofaveron.blogspot. com/2013/07/los-pendejos-y-loscojudos-proposito-de.html

González Castro, J.L. (2006). Memory, narratives and identity. How people recall, transmit and live through historical events. Psicología Política, 32, 7-14.

Halbwachs, M. (1950/1992). On collective memory. Chicago: University of Chicago Press.

Halbwachs, M. (1980). The collective memory. New York: Harper \& Row Colophon Books.

Hampe, T. (2005). La caída del imperio Inca y el surgimiento de la colonia (6-11). En C. Arias (Editor). Compendio Histórico del Perú. (Vol. I., $3^{\text {a }}$ edición). Lima: Milla Batres.

Herranz, J. \& Basabe, N. (1999). Identidad nacional, ideología política y memoria colectiva. Psicología Política, 18, 31- 47.

Hirst, W. \& Manier, D. (2008). Towards a psychology of collective memory. Memory, 16 (3), 183-200.

Hobsbawm, E. \& Ranger, T. (1983). The invention of tradition. Cambridge: Cambridge University Press.

Hogg, M. \& Abrams. D. (1988). Social identifications: a social psychology of intergroup relations and group processes. London: Routledge.

Hogg, M. \& Ridgeway, C. (2003). Social identity: Sociological and social psychological perspectives. Social Psychology Quarterly, 66, 97-100.

Liu, J. \& László, J. (2007). A narrative theory of history and identity: Social identity, social representations, society and the individual. En G. Monloney y I. Walke (eds.), Social representations and Identity: Content, process and power (pp. 85107). London: Palgrave Macmillan.

Liu, J., Goldstein-Hawes, R., Hilton, D., Huang, L., Gastardo-Conaco, C., Dresler Hawke, E. et al. (2005). Social representations of events and people in world history across 12 cultures. Journal of Cross-Cultural Psychology, 36(2), 1-21.

Lyons, E. (1996). Coping with social change: Processes of social memory in the reconstruction of identities. En: Changing European identities: social psychological analyses of social change (pp. 31-40). Oxford: Butterworth-Heinemann.

Ministerio de Defensa. (2014a). Asuntos Históricos: Efemérides Nacionales e Institucionales. Alto Cenepa. Recuperado de http://www. mindef.gob.pe/vercontenido. php? archivo $=$ menu/historicos $/$ 
efem-nacional/efeme-febrero 1. htm\&retorno $=$ si

Ministerio de Defensa. (2014b). Asuntos Históricos: Héroes nacionales. Recuperado de http://www. mindef.gob.pe/vercontenido. $\mathrm{php} ? \operatorname{archivo}=\mathrm{menu} /$ historicos $/$ quinones.htm\&retorno $=$ si

Nigbur, D. \& Cinnirella, M. (2007). National identification, type and specificity of comparison and their effects on descriptions of national character. European Journal of Social Psychology 37, 672-691.

Páez, D., Marques, J., Valencia, J. \& Vincze, O. (2006). Dealing with colective shame and guilt. Psicología Política, 32, 59-78.

Páez, D., Techio, E., Marques, J. \& Martín Beristain, C. (2007). Memoria colectiva y social. En J. Morales, E. Gaviria, M. Moya \& I. Cuadrado (Eds) .Psicología Social. $\quad\left(3^{\mathrm{a}}\right.$ edición). Madrid: McGraw-Hill, 693-716.

Pennebaker, J.W., Páez, D., Deschamps, J-C. et al., (2006). The social psychology of History. Defining the most important events of the last 10, 100 and 1000 years. Psicología Política, 32, 15-32.

Portocarrero, G. \& Oliart, P. (1989). El Perú desde la escuela. Lima: Instituto de Apoyo Agrario.

Quiroz, A. (2013). Historia de la corrupción en el Perú. $2^{\mathrm{a}}$ ed. Lima: IEP.

Rottenbacher, J.M. \& Espinosa, A. (2010). Identidad nacional y memoria histórica colectiva en el Perú. Revista de Psicología 28, 1, 147174.
Sibley, C., Liu, J., Duckitt, J. \& Khan, S. (2008). Social representations of history and the legitimation of social inequality: The form and function of historical negation. European Journal of Social Psychology, 38, 542-565.

Smith, P., Giannini, M., Helkama, K., Maczynski, J. \& Stumpf, C. (2005). Positive autosterotyping and Selfconstrual as predictors of national identification. International Review of Social Psychology, 18, 65-90.

Sosa, F. M., Delfino, G. I., Bobowik, M., \& Zubieta, E. M. (2016). Representaciones sociales de la historia universal: posicionamientos diferenciales en función de la ideología política, religiosidad $\mathrm{y}$ nacionalismo en una muestra argentina. Revista Colombiana de Psicología, 25(1), 47-62. doi: 10.15446/rcp.v25n1.45494

Tajfel, H. (1982). Social psychology of intergroup relations. Annual Review of Psychology, 33, 1-39.

Tajfel, H. (1984). Grupos humanos y categorías sociales: estudios de psicología social. Barcelona: Herder.

Theidon, K. (2009) Entre prójimos: El conflicto armado interno y la política de reconciliación en el Perú. Lima: IEP.

Ucceli, F., Agüero, J., Pease, M.A., Portugal, T. \& Del Pino, P. (2013). Secretos a voces. Memoria y educación en colegios públicos de Lima y Ayacucho. Lima: IEP.

UNESCO (2010). Datos mundiales de educación Perú (7ma edición). 
Memoria de eventos y personajes históricos y su relación con la identidad nacional...

UNESCO. http://www.ibe.unesco. org/fileadmin/user_upload/ Publications/WDE/2010/pdfversions/Peru.pdf

Valencia, J., Espinosa, A., Jiménez, V. \& Romero, J.C. (2018). Ideología, identidad Nacional, representaciones estereotípicas y valoración exogrupal: Un estudio sobre las relaciones entre Perú y Chile. Interciencia, 43(5), 304-312. Vignoles, V., Golledge, J., Regalia, C., Manzi, C. \& Scabini, E. (2006). Beyond Self-Esteem: Influence of multiple motives on identity construction. Journal of Personality and Social Psychology, 90(2), 308-333. 